\title{
Research on Perception Diversity in Intercultural Communication —With Guasha Treatment as a Case Study
}

\author{
Qiong $\mathrm{Ou}$ \\ School of Foreign Languages, Huizhou University, Guangdong 516007, China
}

\begin{abstract}
This paper aims at depicting the phenomenon of perception diversity and its negative impact on intercultural communication (hereafter referred to as IC), as well as trying to offer a corresponding solution. Perception, according to its definition, is a process by which we make what we sense into a meaningful experience by selecting, categorizing and interpreting internal and external stimuli to form our worldviews. Guasha Treatment, a movie which provides the readers with a real intercultural context, is regarded as a classic in English learning and intercultural communication study. With detailed analysis, this paper illustrates that perception diversity will hinder successful IC by causing misunderstandings in communication. Then, based on Berger and Calabrese' Uncertainty Reduction Theory and Gudykunst's Anxiety/Uncertainty Management Theory, empathy is chosen as a solution to reduce the negative impact perception diversity has on IC.
\end{abstract}

Index Terms — perception diversity; intercultural communication; empathy

\section{DEFINITION OF PERCEPTION AND ITS DiverSITY}

In intercultural communication studies, a lot of researchers and scholars have given definitions or description of perception. Perception is, as Marshall Singer (1987) tells us, "the process by which an individual selects, evaluates, and organizes stimuli from the external world" (p.9). Perception, according to De Fleur, Kearney, and Plax (1993), is "seeing, hearing, or feeling something (with the senses) and then identifying what it is within the interpretations learned from one's language and culture" (p.19). Jerome Kagan and Ernest Havemann (1968) define perception as "the process through which we become aware of our environment by organizing and interpreting the evidence of our senses" (p.154). And according to Gamble and Gamble's definition (1996), "Perception is the process of selecting, organizing, and interpreting sensory data in a way that enables people to make sense of our world." (p.77)

From these definitions, we can understand that perception is a process by which we make what we sense into a meaningful experience by selecting, organizing, and interpreting internal and external stimuli. Internal stimuli refer to our needs, desires, interests, motivations and so on. External stimuli are the sensations that arise from the way we see, smell, hear, touch, or taste, etc.

It is easy to notice that people from different cultural backgrounds tend to have different perceptions towards a certain thing or phenomenon, just as K.S. Sitaram and Roy T. Cogdell remarked,

"Members of different cultures look differently at the world around them. Some believe that the physical world is real. Others believe that it is just an illusion. Some believe everything around them is permanent while others say it is transient. Reality is not the same for all people." (qtd. in Samovar et al., 1981, p.106).

American anthropologist Edward T. Hall also introduced to us the concept of perception differences:

"The concept that no two people see exactly the same thing when actively using their eyes in a natural situation is shocking to some people because it implies that not all men relate to the world around them in the same way. Without recognizing these differences, however, the process of translating from one perceptual world to another cannot take place. Significant evidence that people brought up in different cultures live in different perceptual worlds is to be found in the way they orient themselves in space, how they get around and move from one place to the next" (qtd. in Davis, 2001, p.118).

Because of the existence of perception diversity, communicators may encounter misunderstandings in their intercultural interactions, namely, perception diversity has the potential to initiate great obstacles in IC. That is why we probe into the phenomenon of perception diversity in this paper.

During our communication process, it is easy to find perception differences which are determined by one's cultural factors. We may notice that different people sense the outside world differently and they have different interpretations of the same perceived reality. A full moon, to our Chinese people is usually taken to resemble the reunion of family members and often arouses feelings of togetherness or homesickness, but to westerners, it is nothing more than a beautiful physical object. Differences are also to be found in the perception of colors, numbers, animals and many other things. The number 13 in the west is really a taboo, because it means betrayal. So you may notice that even today in the 
west, a large number of buildings don't have the $13^{\text {th }}$ floor. Likewise, the number 4 in China is regarded as an unlucky word and will arouse unpleasant feelings because it has the same pronunciation of the Chinese character si, which means death. Consequently, most Chinese people often avoid choosing the telephone or car number with 4 .

Communicators in intercultural context usually encounter people who do not share their perceptions of the external world, and who may respond to the world in a manner which is often hard to understand. People in England are told by their culture that it is of good manners not to speak too much to strangers. However, in other cultures, such as Arab, Jewish and Italian, where aggression during interaction is considered normal and usually perceived as highly desirable, and these manners are always perceived as non-aggressive behaviors. Therefore, a reserved attitude by English people may be regarded by others as a sign of bad manners. Also, our nonverbal actions are perceived differently from one culture to another. In the US, it is not uncommon to see men walking in the streets or eating in public places without their skirts, but in Hong Kong, skirts are never taken off in public places, because a semi-dressed person in public is regarded as uncivilized. So the meaning attributed to a shirtless person is quite different in Hong Kong than that in the US.

\section{LITERATURE REVIEW}

Researchers who attach importance to perception are mainly scholars or experts in IC or anthropology, such as Larry A. Samovar, Richard E. Porter, William J. Starosta, Chen Guoming, William B. Gudykunst, and Fred E.Jandt, etc. They have given definition to perception, described the perception process, and analyzed its properties of partiality and diversity. Chen Guoming and William J. Starosta (2007) probe into the nature of perception and analyze the stages of perception process. According to their study, the subjective nature of perceptual framework arising from culture usually results in the perception partiality and inaccuracy. Iris Varner and Linda Beamer (2006) argue that "When a receiver perceives those signals, decides to pay attention to them as meaningful, categorizes them according to categories in his or her mind, and finally assigns meaning to them, communication occurs" (p.28). Their study makes us clear about the relationship between perception and communication, namely, communication is the perception of verbal and nonverbal behaviors and the process of assigning meaning to them. And in Communication between Cultures, Larry A. Samovar, Richard E. Porter and Lisa A. Stefani (2000) hold that culture, perception and behavior are directly linked, and differences in cultural beliefs, values and patterns have great influence on perception and communication. William B. Gudykunst and Young Yun Kim (2007) propose that there are three critical aspects of perceptions influencing our communication with strangers: perceptions are selective; perceptions involve categorizations; and rigid categories inhibit effective communication. They analyze that if communicators are mindful enough, they can increase the accuracy of perceptions and attributions about strangers' behavior. Carley H. Dodd (2006) holds that language functions as a filter which molds perception. He deems that our cultural experiences and our language can shape how we see the things around us. David Katan (2004) provides that there are four filters that affect perception of reality: physiological, cultural, individual and language filters. And because of these four filters, the surrounding is not perceived as what "objectively exists in the outside world". Jandt (1995) explains how culture affects the perception process and the way we sense the world. And he comments that perceptional interpretations can reveal much about the culture.

In China, only a few scholars and authors have researched into perception till the present. In their papers, it is acknowledged that perception can affect meaning in communication, thus having a great impact on IC. Ren (2004) sets forth that communicators usually have perception differences because of their cultural backgrounds and these differences usually result in communication misunderstandings. He introduces the mechanism by which culture influences perception, puts forward the concept of cross-cultural perception competence, and also provides strategies to enhance the perception competence of communicators. Cai (1997) contends that without the consideration of cultural perception, it is inappropriate to discuss the meaning of word. Word meanings vary, because people from different cultures make different interpretations of the same word. He also makes clear that prejudices as well as stereotypes will hinder the selection stage of people's perception process, causing failures in IC. By probing into the superficial and deep cultural differences, Guo (2002) provides that it is necessary for communicators to possess the language skills, and pay special attention to cultural perception competence. Li and Wu (2005) suggest that with Hofstede's cultural dimensions as an analytic framework, people can learn how to improve their cultural perception competence, and reduce or eliminate barriers in intercultural communication.

\section{PERCEPTION Diversity AND INTERCULTURAL COMMUNICATION}

Communication is actually the process of our perceiving verbal and nonverbal behaviors and assigning meaning to them. When we perceive some stimuli from the outside environment, we decide to pay attention to them as something meaningful, organize them into categories according to our past experiences, and finally assign meaning to them. Through this process communication has occurred. At any of the three stages of perception, communication can falter even when it takes place between members with very similar values, beliefs, or worldviews, let alone when it occurs between communicators who come from different cultural backgrounds with quite different values or social attitudes. That is why IC can be greatly influenced and hindered with the existence of perception differences. 
In this part we will focus on how perception diversity influences our IC process. In order to achieve a better understanding, the following IC model is employed to make clear how perception diversity causes misunderstandings:

Culture A Culture B
A Conder $\rightarrow$ encoding $\rightarrow$ message $\rightarrow$ decoding $\rightarrow$ receiver
Misunderstandings
spective.
eiver decodes the message depending on the perception from his $/$ her
perspective.

Here goes a brief introduction to the model: the sender and the receiver come from different cultures: culture A and culture B. In their IC interaction, when the sender encounters some outside stimuli, he/she will encode these stimuli into a message and send it to the receiver. On the other hand, the receiver gets the message and decodes it in order to understand his/her counterpart's intention. (1) and (2) represents two assumptions.

(1) refers to the assumption that the sender encodes a message depending on the perception from his/her own cultural perspective. (2) refers to the assumption that the receiver decodes the message depending on the perception from his/her own cultural perspective. Mainly due to their cultural differences, the sender and the receiver tend to have different perceptions of the outside stimuli, hence perception diversity. If the sender encodes the message and the receiver decodes the message both according to the perception from his/her own cultural perspective, with such perception diversity, a message decoded by the receiver may be different from what is encoded by the sender. Therefore, misunderstandings emerge, causing problems in IC.

\section{B. Misunderstandings Caused by Perception Diversity in IC from the Movie Guasha Treatment}

In IC, we will meet with a lot of communication misunderstandings or even failures because of perception diversity, so understanding and appreciating differences in perception are crucial if we are to improve our ability to get along with people from other cultures. Here we will take some examples of different perceptions among cultures with the purpose to explain that perception is culturally-determined and that perception diversity may cause serious IC misunderstandings.

With the aim of giving a vivid account of perception diversity and positioning readers in a real intercultural interaction context, we choose the movie Guasha Treatment, a movie widely accepted as a classic for displaying culture shock, for analysis. In the following, with an in-depth analysis of perception differences in the movie, it is easy to draw a conclusion that misunderstandings or conflicts in IC can be attributed to perception diversity. Differences in perception reflected in the movie mainly come from the following aspects:

1. Different Perceptions of the Practice of Guasha Treatment

The main conflict in the movie is triggered by guasha treatment. Senior $\mathrm{Xu}$ (the grandfather) gives Dennis (the grandson) a guasha treatment which causes terrible scars on Dennis's back. When the scars are examined by doctors, they regard Dennis is suffering child abuse and severe neglect at home, so they send him to Child Welfare Agency for special care. But as a matter of fact, guasha treatment is an ancient oriental medical practice that can be used to release toxins from the body with jade or horn blade or finger at relevant acupuncture points by scraping, pinching, pressing, lifting, or puncturing the skin. Most Chinese people take it as a family remedy. In the movie, Senior Xu does guasha to his grandson and intends to cure the latter's cold. While American doctors, viewing the scars left by guasha on Dennis's back, deem that he lives in a very dangerous home environment and should be kept under the protection of the state government. This kind of conflict arises due to the different western and eastern medical treatment principles, based on people's different perceptions of the relationship between man and nature. To the Chinese, guasha treatment is an assistant treatment of Traditional Chinese Medicine, representing the Chinese philosophy of wholeness of man and nature - the ideal harmony, aims at keeping the balance of qi, blood, yin and yang through meridians and collaterals so as to adjust the relationship between human body and nature. This kind of medical treatment principle, rather experiential, individual and ambiguous, turns out to be too hard to describe and understand for foreigners. On the contrary, the western medical treatment principle, which concentrates on clarity and confirmation, is used by most western people to view the whole world by microcosmic analyzing, and objective verifying. According to this principle, modern westerners regard human body composed of independent parts, and disease is a partial trauma of certain organ. So in their opinion, guasha treatment is unworkable in curing a cold and it is nothing but a kind of body abuse. 
2. Different Perceptions of the Relationship between Father and Son

Chinese relationship between father and son is unequal, which can be seen from Xu Datong (Dennis's father)'s willingness to be the scapegoat. In fact, it is Senior Xu who does guasha treatment on Dennis, but when the judge asks Xu Datong, "Did you do this guasha treatment to your son yourself?" Datong answers "yes" without hesitation. Datong does so because he doesn't want his father to be in trouble. Datong is willing to be the scapegoat and admits that it is him rather than his father who does guasha treatment to his son, which is a manifestation of filial sacrifice. Most westerners, including Datong's boss John can not understand this, because in their eyes, everyone is equal and should be self-responsible for their behavior. Father and son are equal, and if it is father's fault, father should admit it.

Relationships between father and son in America and China are quite different. Among Chinese, the kinship is of great priority and inequality extends into family, particularly into the relationship between father and his children. Father is always accepted as the ruler and master of the family, indicating that he represents authority and occupies high family position. Children have to obey unconditionally father's rules and show great filial piety to parents. Children's relations with father are very formal and love for him is always combined with awe. In America, however, along with the American emphasis on individual freedom, the belief in equality has had a strong effect on family. American families, compared with those in China, emphasize more on their members' independence, freedom and equality. So the relationship between parents and children in western families is more like friends, equal and casual.

Such perception differences can also be used to explain Dennis's rebellion towards his father. Being hit by his father, Dennis cried: "You never hit me before. Dads who hit their kids are not good Daddies." Dennis is brought up in American culture, which emphasizes equality among family members, so he doesn't realize he is challenging his father's authority.

3. Different Perceptions of Face

At the ceremony where Xu Datong is being honored, Dennis hits Paul, John's son. Xu asks Dennis to apologize to Paul, but Dennis refuses. Xu loses his temper and gives Dennis a slap fiercely in the presence of Paul's father. Xu disciplines his son in public, and the following is the conversation between $\mathrm{Xu}$ and John on this matter.

Xu: "Why I hit him?

Why? ...To give you face, you know?

He is my own son. I hit him to show my respect for you."

John: "What kind a twist Chinese logic is that?

You have hit your own son so you can show your respect to me?"

Dennis hits Paul and refuses to apologize, and in Xu's opinion, this behavior is violating John's face, so Xu punishes his son to show his respect to his boss. But this logic is irrational and unacceptable to Americans, because their culture emphasizes equality among people, including father and son and it is regarded wrong for father to hit son in public. The main reason for this disagreement is that Xu and John, representatives of Chinese and American cultures respectively, perceive "face" differently. Chinese people value face very much, and in their daily life, they will try all means to save face and avoid negative remarks or behaviors which violate others' face, because Chinese people value harmonious relationship highly, want to avoid conflicts, and protect the integrity of group. Consequently, face-saving behaviors take on great significance.

Additionally, in the movie, according to Xu Datong, hitting his son is not only an act to show respect to his boss, but also a means to save his own face. Dennis does not obey Xu's order to apologize to Paul, which is regarded as a challenge to the father's authority, especially in public. Son shouldn't violate his father's will and if he does, then the father's disciplining his son is definitely right and appropriate, because it is a way of educating children in China. But American people can not understand this notion of face and the logic of giving somebody face by father's disciplining his son. That's why John remarked "What kind a twist Chinese logic is that?"

4. Different Perceptions of Abortion

Mrs. Rico, the nurse who assists in Xu's wife giving birth to Dennis, accuses Xu Datong of giving instructions to kill his child in order to save his wife's life, while Xu thinks it is human's common sense. This argument shows people's different perceptions of abortion. In western countries, where equality is highly valued, abortion is regarded as an unfair and inhumane act, which means taking away others' lives. The Catholic Church, as well as the majority of society, condemning abortion as a grave evil, is extremely against it. But in Xu and most Chinese's opinions, people should spare no efforts to save the adults' lives first. Abortion in China has not caused much debate about killing human beings; instead, it is sometimes taken as a means by women to complement the government's family-planning policy, aiming at controlling China's large popularity.

\section{EMPATHY AS A CORRESPONDING SOLUTION}

\section{A. Berger and Calabrese's Uncertainty Reduction Theory}

Berger (1979) and Berger and Calabrese (1975) developed uncertainty reduction theory (hereafter referred to as the URT) to probe into the ways people come to know each other in their budding relationship development. Uncertainty refers to people's cognitive inability to explain feelings and behaviors of themselves or others in interactions owing to ambiguous situations that trigger anxiety. Berger and Calabrese (1975) summarize this theory: "Central to the present theory is the assumption that when strangers meet, their primary concern is one of uncertainty reduction or increasing 
predictability about the behavior of both themselves and others in the interaction" ( p. 100). According to this theory, in our interpersonal encounters, we meet with ambiguity or uncertainty about relationships and experience discomfort with questions about relationships. In order to make successful IC, communicators may have to engage in behaviors that can enhance the chances of maximum understanding, and reduce uncertainty as well as discomfort. That is to say, the URT informs us that interpersonal relationship will develop and progress if communicators are able to reduce uncertainty about one another.

\section{B. Gudykunst's Anxiety/Uncertainty Management Theory}

Gudykunst and Hammer (1988) used uncertainty and anxiety to explain intercultural adjustment. By extending Berger and Calabrese's uncertainty reduction theory, Gudykunst develops anxiety/uncertainty management theory (hereafter referred to as the AUM theory), a theory based on the assumption that managing uncertainty and anxiety is necessary and sufficient for effective communication and intercultural adjustment.

According to the AUM theory, when we communicate with strangers, especially those from another culture, we have not only a high level of uncertainty, but also a high level of anxiety. If uncertainty and anxiety levels are too high, we can not interpret others' messages accurately or predict their behaviors appropriately. But if we learn much of the strangers' culture, the more we know it, the less uncertainty and anxiety we will encounter, and the higher attribution confidence we will possess. With the reduction in uncertainty and anxiety and enhancement of attribution confidence, communicators will display more certainty and confidence in the process of attributing meanings to the selected stimuli, as well as get a precise and correct understanding of them, therefore, effective IC will be achieved. Concerning the importance of reducing uncertainty and anxiety, Gudykunst's AUM theory offers a powerful support with the Axiom 39:

An increase in our ability to manage our anxiety about interacting with strangers and an increase in the accuracy of our predictions and explanations regarding their behavior will produce an increase in the effectiveness of our communication. (Gudykunst, 1977, p.307)

To be brief, our ability to communicate effectively is based, at least partially, on our ability to manage our anxiety and uncertainty.

The above presentation of the URT and the AUM Theory inform us that, along with communication between people from different cultures, there are always uncertainty and anxiety. A high level of uncertainty and anxiety may cause communicators' inability to interpret others' messages, which inevitably hinders the success of IC. Therefore, to reduce or manage uncertainty and anxiety becomes a common need of all communicators, as well as a guarantee of successful IC.

In order to reduce uncertainty/anxiety, and achieve effective IC, communicators will take one of the two options to interpret the unknown or strange, i.e. to perceive the outside stimuli from their own cultural perspective, or from their counterparts' cultural perspective. With the analysis of the IC model employed in this paper, we are clear that: in IC, if the sender and the receiver try to reduce uncertainty and anxiety by drawing on perceptions from their respective cultural perspectives, owing to perception differences, the message decoded will differ from the one that is encoded, thus misunderstandings appearing. However, if either the sender or the receiver chooses to reduce uncertainty and anxiety by perceiving the outside from his/her counterpart's cultural perspective, because of similar perceptions, the message decoded by the receiver will be similar to the one that is encoded by the sender. In this way, mutual understanding will be achieved and smooth IC will be guaranteed.

Conclusively, in order to reduce uncertainty and anxiety in IC, communicators are advised to encode or decode a message according to the perception from their counterparts', rather than their own cultural perspectives. And to perceive from their counterparts' cultural perspectives requires empathy. That is why empathy is put forward as a corresponding solution to eliminate or reduce the negative impact perception diversity has on IC. Namely, if the sender or the receiver can empathize and perceive the outside from his/her counterpart's cultural perspective, the message decoded will be similar to what is encoded, misunderstandings will not arise, and the negative impact of perception diversity on IC which is mentioned before will be eliminated or reduced.

\section{The Importance of Empathy in IC}

One skill that consistently emerges in the discussion of effective communication with strangers is empathy. Communication, especially IC, needs empathy to a greater extent. Empathy is "the ability to feel like another or to place oneself in another's shoes" (Combs and Snygg, 1959, p.234-236). In other words, it refers to the ability to view a situation from another's point of view. It is the process of reaching beyond the self and understanding and feeling what another person understands or feels.

Many scholars have explored the effectiveness of empathy in intercultural interactions. Most of them admit that empathy is one of the determinants of intercultural competence. Broome sees empathy as "important to both general communication competence and as a central characteristic of competent and effective IC" (qtd. in Samover et al.,_2000, p. 259). Samover (2000) also remarks "to be successful as an intercultural communicator, you must develop empathy" (p. 261). Showing empathy is an important factor in maintaining the ongoing conversation and achieving the successful communication. To display empathy, that is to say, to enjoy the ability to see things from others' point of view is a critical skill for the success of IC. "Empathic persons know how to show understanding by projecting themselves into their partner's position" (Chen and Starosta, 2007, p. 42). It is acknowledged that partners characterized by high levels 
of warmth and compassion, and showing more empathic concerns to others are especially valued as effective communicators and therefore prompt more communication from others. Thus empathy is the starting point of an effective intercultural and interpersonal communication climate which lays the firm foundation for adjusting the cultural differences and avoiding misunderstanding. And "A lack of empathy always represents a potential communication problem. Our inability to understand and appreciate the point of view and life orientation of others often keeps us from effectively communicating with them" (Samovar et al., 1981, p. 209).

\section{The Application of Empathy in This Study}

In the following, we try to illustrate how empathy can be used to reduce the negative impact perception diversity has on IC. From the IC model, we are clear that encoding and decoding are two important processes. Usually, the sender encodes a message according to what he/she perceives, and assumes that his/her counterpart will absolutely understand the message by decoding it with similar perception. However, due to the cultural influence, people tend to have different perceptions of the same stimulus. Therefore, the message decoded by the receiver will possibly be different from that encoded by the sender. In this way, misunderstandings arise and IC becomes difficult. However, if the sender or the receiver chooses to perceive the outside from the other part's cultural perspective, they will have similar interpretations, and then, the message decoded will be similar to the one that is encoded, so misunderstandings will be avoided. Therefore we suggest taking empathy as a solution to avoid misunderstandings caused by perception diversity so as to achieve desirable communication results. In the following, the examination of empathy in the processes of encoding and decoding will help us see how it can be applied in IC. Encoding refers to the process that the sender, after perceiving the outside stimuli, puts them into a message; while decoding is the process that the receiver interprets the meaning of the message encoded by the sender. Communication breakdowns usually occur if the receiver cannot decode what has been encoded in the message, and this is particularly true with IC due to the existence of perception diversity.

1. Empathy Being Applied to the Encoding Process

From the IC model, if the sender encodes a message depending on the perception from his/her own cultural perspective, because of the existence of perception diversity, the message encoded will be difficult for the receiver to understand. In order to guarantee smooth IC, we should take empathy into the process of encoding, which means, to encode a message according to the perception from the counterpart's cultural perspective.

For instance, people in China often greet each other with such sentences: "Where are you going?" or "What are you doing?" These sentences are not intentionally inquiring about others' private life; instead, they are only used for greeting or showing one's care and consideration, indicating an intimate human relationship. However, foreigners, who focus much on privacy, upon hearing these sentences, will feel uncomfortable, embarrassed or even irritated because they feel their personal life has been disturbed. Under this circumstance, by applying empathy and changing the sentences into "How do you do?", "It is a nice day, isn't it?" or just "Hello!" we can send the accurate information of greeting others. In this way, both the sender and the receiver can understand each other's true intention and get smooth IC.

2. Empathy Being Applied to the Decoding Process

Decoding refers to the receiver's interpreting the message encoded by the sender. If the receiver can not decode what has been encoded in the message, a communication failure emerges. For example, when Chinese people, especially those in the north meet each other, they are accustomed to greet each other by asking "Have you had your lunch?", a sentence has nothing to do with sending an invitation. However, without the knowledge of this custom, the foreigners, when decoding the message, will perceive it in their own way-such a sentence in their own language infers an invitation for dinner. Thus misunderstanding occurs.

Similarly, Chinese people's modesty usually constitutes something difficult for foreigners to understand. In China, people are told by their culture to be modest and self-dispraising. So when entertaining friends at dinner, as a reply to others' compliments on the dishes, the host will always say: "There is no delicious food, and the condition is so poor. Please forgive me!" It is because "to Chinese, however, the customary reply to a compliment would be to claim that one is not worthy of the praise, that what one has done is not enough....and acceptance of compliment would imply conceit or lack of manners" (Deng and Liu, 2003, p. 2). If the foreigner friends do not know the Chinese culture and decode the message with the perception from their own cultural perspective, they will get embarrassed, feeling not being treated well. But if the foreign friends employ empathy in decoding this message, i.e. to decode the message with the perception from the Chinese cultural perspective, they will understand that such sentences do not mean inhospitality, but merely a sign of showing modesty. Therefore, knowing cultural differences and decode the messages with the perception from the counterpart's perspective is important for people to understand what is actually being encoded.

\section{CONCLUSION}

This paper has proposed a new approach to the IC study - from the perspective of perception. It tries to make such a point clear: studies on perception diversity are of great importance in IC. Due to the cultural influence, people in the world have different perceptions and these perception differences will initiate obstacles in understanding, thus making effective IC hard to achieve. The main purpose of this paper is to elaborate the negative impact perception diversity has on IC, as well as try to offer a corresponding solution. 
In order to illustrate the negative impact perception diversity has on IC, we employ an IC model, as well as the movie Guasha Treatment. With the description of an IC model, readers can understand how on earth perception diversity causes misunderstandings in IC. And the movie Guasha Treatment, a classic movie mainly about culture shock, positions readers in a real intercultural context and makes them clear that a lot of IC misunderstandings or failures are triggered by people's different perceptions.

Finally, in order to solve the problems caused by perception diversity in IC, empathy is chosen as a sound solution. According to the URT and the AUM Theory, in communication, it is human being's common need to reduce uncertainty and anxiety for the sake of guaranteeing desirable communication outcome. To reduce uncertainty and anxiety, communicators have to take one of the two options: to perceive the outside stimuli from the cultural perspective of themselves or from the cultural perspective of their counterparts. From the IC model employed in this paper, we are clear that if the sender encodes the message and the receiver decodes the message both according to the perception from their respective cultural perspectives, because of their different perceptions, the message decoded will be quite different from the one that is encoded, misunderstandings arise and communication usually fails. Instead, if either the sender or the receiver can apply empathy, encodes or decodes the message with the perception from the counterpart's perspective, the message decoded will be similar to the one that is encoded, and it would be easier for them to achieve success in IC.

\section{REFERENCES}

[1] Berger, C. R. (1979). Beyond Initial Interactions: Uncertainty, Understanding, and the Development of Interpersonal Relationships. In Giles, H., and Clair, R. St. (Eds.) Language and Social Psychology. Oxford: Basil Blackwell, 122-144.

[2] Berger, C. \& Calabrese, R. (1975). Some Explorations in Initial Interactions and Beyond: Toward a Developmental Theory of Interpersonal Communication. HumanCommunication Research, 1, 99-112.

[3] Chen Guo-Ming \& Starosta, W. J. (2007). Foundations of Intercultural Communication. Shanghai: Shanghai Foreign Language Education Press.

[4] Cai Jianping. (1997). The Influence of Cultural Perception on Word Meanings. Foreign Languages and Their Teaching, 3 , 19-21.

[5] Combs, A. \& Snygg, D. (1959). Individual Behavior: A Perceptual Approach to Behavior. New York: Harper \& Row.

[6] Dodd, C. H. (2006). Dynamics of Intercultural Communication ( $5^{\text {th }}$ ed.). Shanghai: Shanghai Foreign Language Education Press, 128-129.

[7] Davis, L. (2001). Doing Culture: Cross-cultural Communication in Action. Beijing: Foreign Language Teaching and Research Press.

[8] De Fleur, M.L., Kearney, P., \& Plax, T. G. (1993). Fundamentals of Human Communication Mountain View. CA: Mayfield.

[9] Deng Yanchang \& Liu Runqing. (2003). Culture and Language. Beijing: Foreign Language Teaching and Research Press.

[10] Guo Shuping. (2002). The Cultural Perception and Memory in Intercultural Communication. Henan Social Sciences, 4,101-102.

[11] Gamble, T. K. \& Gamble, M. (1996). Communication Works (5 ${ }^{\text {th }}$ ed.). New York: McGraw-Hill.

[12] Gudykunst, W. B. (1977). Theorizing about Intercultural Communication. Thousand Oaks, CA: Sage.

[13] Gudykunst, W. B. \& Hammer, M. R. (1988). Strangers and Hosts: An Uncertainty Reduction Based Theory of Intercultural Adaptation. In Kim, Y. Y. and Gudykunst, W. B. (Eds.) Cross-cultural Adaptation: Current Approaches. Newbury Park, CA: Sage, 106-139.

[14] Gudykunst, W. B. \& Kim, Y. Y. (2007). Communicating with Strangers: An Approach to Intercultural Communication (4 ${ }^{\text {th }}$ ed.). Shanghai: Shanghai Foreign Language Education Press.

[15] Jandt, F. E. (1995). Intercultural Communication: An Introduction. Thousand Oaks, CA: Sage.

[16] Katan, D. (2004). Translating Across Culture: An Introduction for Translators, Interpreters and Mediators. Shanghai: Shanghai Foreign Language Education Press, 86-87.

[17] Kagan, J. \& Havemann, E. (1968). Psychology: An Introduction. New York: Harcourt Brace Jovanovich.

[18] Li Xiaohong \&Wu Xiaochuang. (2005). Communication Barriers and Cultural Perception in Intercultural Communication. Seeker, 5, 70-72.

[19] Ren Yuhai. (2004).The Developing Strategy for Cross-cultural Perception Competence. Culture Research, 12, 52-65.

[20] Singer, M. (1987). Intercultural Communication: A Perceptual Approach. Englewood Cliffs, NJ: Prentice-Hall.

[21] Samovar, L. A. \& Porter, R. E. (1981). Understanding Intercultural Communication. Belmont, CA: Wadsworth Publishing Company.

[22] Samovar, L. A., Porter, R. E. \& Jain, N. C. (1981). Understanding Intercultural Communication. Belmont, CA: Wadsworth Publishing Company.

[23] Samovar, L. A., Porter, R. E. \& Stefani, L.A. (2000). Communication between Cultures (3 ${ }^{\text {rd }}$ ed.). Beijing: Foreign language Teaching and Research Press.

[24] Varner I. \& Beamer L. (2006). Intercultural Communication in the Global Workplace (3 ${ }^{\text {rd }}$ ed.). Shanghai: Shanghai Foreign Language Education Press.

Qiong Ou was born in Hunan, China in 1981. She received her Master's degree in English Language and Literature from Xiangtan University, China in 2010. She is currently a lecturer in the School of Foreign Languages, Huizhou University, Guangdong, China. Her research interests include cross-cultural communication and pragmatics. 